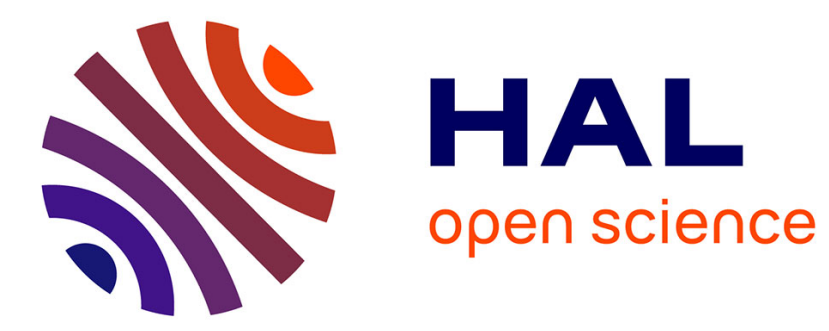

\title{
Possibilités et limites de l'utilisation du verrat en accouplement naturel
}

\author{
Raymond Nowak, M. Paquignon, J.P. Signoret
}

\section{To cite this version:}

Raymond Nowak, M. Paquignon, J.P. Signoret. Possibilités et limites de l'utilisation du verrat en accouplement naturel. Productions Animales, 1988, 1 (3), pp.215-218. hal-00895835

\section{HAL Id: hal-00895835 \\ https://hal.science/hal-00895835}

Submitted on 1 Jan 1988

HAL is a multi-disciplinary open access archive for the deposit and dissemination of scientific research documents, whether they are published or not. The documents may come from teaching and research institutions in France or abroad, or from public or private research centers.
L'archive ouverte pluridisciplinaire HAL, est destinée au dépôt et à la diffusion de documents scientifiques de niveau recherche, publiés ou non, émanant des établissements d'enseignement et de recherche français ou étrangers, des laboratoires publics ou privés. 
INRA Prod. Anim., 1988, 1 (3), 215-218

\section{R. NOWAK, M. PAQUIGNON* J.P. SIGNORET}

INRA Nouzilly

Station de Physiologie de la Reproduction 37380 Monnaie

* Institut Technique du Porc

149, rue de Bercy

75595 Paris Cedex 12

\section{Possibilités et limites de l'utilisation du verrat en accouplement naturel}

\section{Malgré la mise au point de techniques efficaces d'insémination artificielle, l'accouplement naturel reste généralement utilisé dans la pratique de l'élevage porcin moderne. Or, le développement de l'élevage des truies en bandes impose au verrat un rythme de saillies élevé, ce qui peut avoir des conséquences sur le pouvoir fécondant du sperme.}

L'utilisation du mâle repose sur ses capacités de comportement sexuel, et sur la notion empirique de « fatigue » du reproducteur. La capacité de fécondation résultant d'un accouplement est le plus souvent considérée comme constante et optimale pourvu que le verrat soit normal.

Or, chez le verrat, l'éjaculation mobilise la plus grande partie des réserves spermatiques épididymaires disponibles. Le nombre de spermatozoïdes émis au cours d'éjaculats successifs diminue ainsi très rapidement (Kaplan 1969).

\section{Résumé}

Quinze verrats Large-White ou Land-Race de 9 mois ont été soumis au rythme de 1 , 2,3 ou 4 éjaculats journaliers pendant 5 jours, suivis d'un repos sexuel de deux semaines. Si tous ont pu réaliser une éjaculation journalière, respectivement $91,6 \%, 58,3 \%$ et $41,6 \%$ d'entre eux ont pu produire du sperme aux autres rythmes.

Le nombre de spermatozoïdes par éjaculat décroît pour atteindre, le cinquième jour, respectivement, environ 15, puis moins de 10 et 5 milliards pour un, deux et quatre éjaculats journaliers. Dans ces deux derniers cas, $12 \%$ et $31 \%$ des éjaculats contenaient moins de 3 milliards de spermatozoïdes, c'est-à-dire le nombre minimum efficace dans le cas de l'utilisation en insémination artificielle.

Pour valider les résultats en accouplement naturel, des truies nullipares ont été abattues immédiatement après la saillie pour mesurer le volume de l'éjaculat et le nombre de spermatozoïdes émis. D'autres femelles ont été sacrifiées 35 jours après l'accouplement pour apprécier la fertilité. Quel que soit le rythme d'éjaculation, le volume et le nombre de spermatozoïdes ne sont pas différents en collecte de sperme ou en accouplement naturel. Par contre, un reflux variable et important réduit le volume de sperme et le nombre de spermatozoïdes effectivement déposés dans l'appareil génital de la femelle.

La fertilité décroit significativement lorsque le rythme d'éjaculation s'élève : elle est de 91,6\% contre 58,3\% lorsque les mâles ont effectué au préalable une ou quatre éjaculations journalières. Ce phènomène semble refléter la réduction du nombre de spermatozoïdes mis en place.
Or les études de la production de spermatozoïdes par le verrat ont toutes été faites dans le but de leur utilisation en insémination artificielle, et avec un rythme d'éjaculation peu élevé. C'est ainsi que le nombre total de spermatozoïdes produit n'est que peu modifié lorsque le nombre de collectes de sperme passe de 1 à 3 par semaine (du Mesnil du Buisson et Signoret 1970). Par contre aucune donnée n'est disponible sur l'évolution de la production spermatique du verrat dans les conditions qui sont celles d'une utilisation intensive en accouplement naturel.

Le but de l'expérimentation que nous avons entreprise était donc de mesurer la production spermatique du verrat soumis à une activité sexuelle intense, d'évaluer les caractéristiques obtenues dans ces conditions en accouplement naturel, et, enfin, d'apprécier d'éventuelles conséquences du rythme d'éjaculation sur la fertilité

\section{1 / Conditions expérimentales}

\section{1 / Animaux}

Quinze verrats de trois souches différentes (5 Large-White "Poitou », 5 Large-White UPRA, 5 Landrace UPRA), nés entre le 11 mars et le 13 avril 1982, et pesant de 108 à $123 \mathrm{~kg}$ en début d'expérimentation, ont été entraînés à la collecte pendant une période de 4 semaines. Les 12 verrats ( 4 de chaque souche) qui se sont montrés les plus aptes à la collecte ont alors été répartis au hasard en trois groupes. Les diverses séries expérimentales ont eu lieu alors que leurs âges étaient compris entre 10 et 20 mois. 
Dix huit jeunes truies nullipares ont été synchronisées par un progestagène de synthèse ("Regumate ", Roussel UCLAF) et utilisées au moment de l'œstrus pour mesurer la quantité de spermatozoïdes déposée dans l'utérus lors de la saillie. Vingt quatre autres ont été utilisées pour apprécier la fertilité.

\section{2 / Modalités d'éjaculation}

Les collectes de sperme ont eu lieu dans le bâtiment où vivaient les animaux. Pendant le déroulement de l'expérience, certains verrats soumis à un rythme élevé d'éjaculations n'ont pas réagi immédiatement au mannequin. Dans ce cas, une truie de petite taille était attachée sous le mannequin pour stimuler leur réaction sexuelle.

Les techniques de collecte et d'examen de la semence sont classiques: volume, concentration, motilité et formes anormales des spermatozoïdes.

Les accouplements ont eu lieu dans une case au sol paillé. Leur observation a montré l'existence d'un reflux de sperme important. Aussi, ces pertes ont-elles été recueillies dans un flacon.

Immédiatement après la fin de l'accouplement, avec un délai moyen de 7 minutes, dixhuit femelles ont été abattues. L'appareil génital était immédiatement prélevé et perfusé avec du liquide physiologique. Le perfusat a été recueilli après filtration pour comptage des spermatozoïdes.

Vingt-quatre autres femelles ont été abattues 35 jours après l'accouplement, le nombre d'embryons et celui des corps jaunes ont été déterminés.

\section{3 / Plan expérimental}

Trois parties ont été envisagées successivement :

\section{a / Effet du rythme d'éjaculation sur la composition de l'éjaculat}

Chaque verrat a été soumis à un rythme de 1 , 2,3 ou 4 collectes journalières pendant 5 jours suivis d'un repos sexuel de deux semaines. Pendant la semaine de collectes, chaque mâle conservait le même rythme d'éjaculation. Toutes les trois semaines, ce rythme était permuté jusqu'à ce que tous les verrats aient subi les différents rythmes.

\section{b / Comparaison de la collecte et de l'accouplement naturel}

Nous n'avons utilisé dans cette seconde phase que les 6 mâles qui avaient pu être utilisés dans la première phase sans avoir refusé plus d'une fois d'éjaculer. Après un repos sexuel de deux semaines, chacun d'entre eux a été soumis au rythme d'éjaculation de 1 ou de 4 fois par jour pendant 5 jours successifs. Après une nouvelle période de repos sexuel de deux semaines, le rythme a été permuté.

Les éjaculations ont été obtenues par collecte sauf à trois moments différents où la collecte a été remplacée par une saillie : le premier éjaculat (premier jour), et le quatrième (quatrième jour), dans le cas du rythme de 1 éjaculat journalier, et le troisième éjaculat de la quatrième journée dans le cas du rythme de quatre éjaculations journalières.

\section{c / Estimation de la fertilité}

Afin d'estimer la fertilité obtenue dans ces conditions, la première et la quatrième collecte au rythme d'une éjaculation par jour ont été remplacées par des saillies naturelles et ce, pour six femelles dans chaque cas. Il en a été de même pour la seconde collecte des $4^{\mathrm{e}}$ et $5^{\mathrm{e}}$ journée au rythme de 4 éjaculats par jour.

\section{2 / Résultats}

\section{1 / Comportement sexuel}

Les réactions sexuelles des verrats deviennent plus lentes lorsque le rythme d'éjaculation augmente. Des refus de monte apparaissent (Tableau1), qui nécessitent l'emploi d'une femelle boute en train. Mais même avec cet artifice, un des mâles finit par refuser de monter après quatre jours d'éjaculations biquotidiennes. Ceci apparaît dès le premier jour lorsque la fréquence d'éjaculation quotidienne est de 3 ou 4 . Ces rythmes de collecte ont pu être maintenus chez seulement la moitié environ des mâles $(58,3 \%$ et $41,6 \%$ respectivement pour les rythmes 3 et 4).

L'irrégularité dans l'intervalle des sollicitations sexuelles influe sur les réponses des mâles : quel que soit le jour, la motivation est plus forte le matin après un repos sexuel de plus de 16 heures.

Tableau 1. Effet de la race du verrat et du rythme quotidien de sollicitation sur le taux de réalisation de l'éjaculation ( $n=4$ verrats par race).

\begin{tabular}{|c|c|c|c|c|}
\hline $\begin{array}{c}\text { Rythme quotidien } \\
\text { d'éjaculation }\end{array}$ & $\begin{array}{c}\text { Large-White } \\
\text { UPRA }\end{array}$ & $\begin{array}{c}\text { Race des verrats } \\
\text { Large-White } \\
\text { Poitou }\end{array}$ & $\begin{array}{c}\text { Land-Race } \\
\text { UPRA }\end{array}$ & Total \\
\hline 1 & 100 & 100 & 100 & 100 \\
2 & 100 & 75 & 100 & 91,6 \\
3 & 75 & 25 & 75 & 58,3 \\
4 & 50 & 0 & 75 & 41,6 \\
\hline
\end{tabular}




\begin{tabular}{|c|c|c|c|c|r|r|}
\hline \multirow{2}{*}{$\begin{array}{c}\text { Rythme quotidien } \\
\text { d'éjaculation }\end{array}$} & $\mathbf{1}$ & $\mathbf{2}$ & $\mathbf{3}$ & $\mathbf{4}$ & $\mathbf{5}$ & \multirow{2}{*}{ Total } \\
\cline { 2 - 6 } & $\mathbf{1}$ & 0 & 0 & 0 & 8,3 & 1,6 \\
$2(12)$ & 0 & 4,1 & 4,1 & 4,1 & 26,1 & 7,5 \\
$3(24)$ & 2,8 & 8,6 & 14,7 & 20,6 & 34,3 & 16,2 \\
$4(48)$ & 2,1 & 12,5 & 28,6 & 38,1 & 44,7 & 24,5 \\
Total & $\mathbf{1 , 7}$ & $\mathbf{8 , 1}$ & $\mathbf{1 5 , 2}$ & 21,4 & 33,3 & 15,9 \\
\hline
\end{tabular}

Tableau 2. Répartition du pourcentage d'éjaculats ayant moins de $3 \times 10^{9}$

spermatozoïdes selon le jour et le rythme de récolte.

Tableau 3. Evolution des pertes en spermatozoïdes au moment de la saillie en fonction du numéro de l'éjaculation dans la semaine.

\section{$2.2 /$ Production de sperme}

\section{a / Collecte}

Le nombre de spermatozoïdes par éjaculat diminue rapidement au cours de collectes successives. Avec une éjaculation journalière, il décroit de 53 à environ 15 milliards le cinquième jour. Lorsque le rythme de collectes journalières est plus élevé, la décroissance du nombre total de spermatozoïdes par éjaculat est encore plus rapide (Figure 1). Dès le troisième jour, le nombre de spermatozoïdes par éjaculation n'excède pas 10 milliards lors d'éjaculations biquotidiennes, et ne dépasse que rarement 5 milliards lorsque le nombre de collectes est de 3 ou 4 par jour.

Si nous considérons que le nombre minimum nécessaire pour obtenir un taux de fécondation optimum est, en insémination artificielle, de 3 milliards (Signoret et al 1971), il est intéressant d'observer l'apparition des éjaculats contenant un nombre de spermatozoïdes inférieur à cette valeur. Nous pouvons, en effet, émettre l'hypothèse que des accouplements correspondants pourraient, de ce fait, aboutir à un taux de fécondation réduit. Ces éjaculats n'apparaissent qu'après le cinquième jour d'activité sexuelle lorsque les éjaculations sont quotidiennes (Tableau2). Par contre, ceci se produit dès le second jour pour des éjaculations biquotidiennes et même le premier jour lorsque le rythme d'activité sexuelle est plus élevé. La fréquence des collectes en augmente régulièrement le nombre au cours des 5 journées d'activité sexuelle.

\section{b / Comparaison de la composition de l'éjaculat obtenu par collecte ou en accouplement}

A cause du reflux de sperme observé durant l'accouplement, le nombre total de spermatozoïdes émis dans l'utérus est toujours plus faible lors d'un accouplement naturel qu'après collecte du sperme. Ces pertes sont d'autant plus élevées que la production de spermatozoïdes est importante. Elles atteignent en moyenne près de la moitié des spermatozoïdes éjaculés. Une grande variabilité est constatée en ce qui concerne l'importance de ces pertes (Tableau 3). La répartition des accouplements selon cette importance (Figure 2) montre deux tendances :
Figure 1. Evolution du nombre de spermatozoïdes par éjaculat en fonction du rythme d'éjaculation.

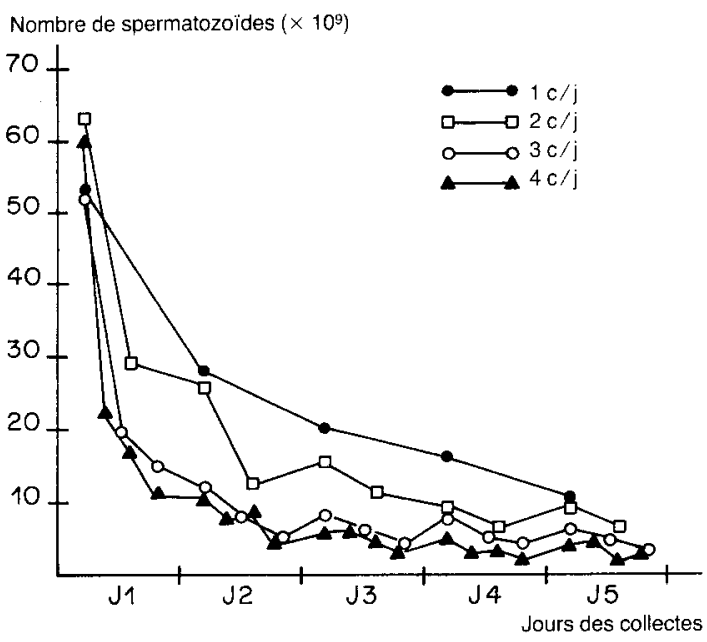

Figure 2. Répartition des saillies en fonction du pourcentage des pertes en sperme (phase liquide) et en spermatozoïdes $(N=16)$.
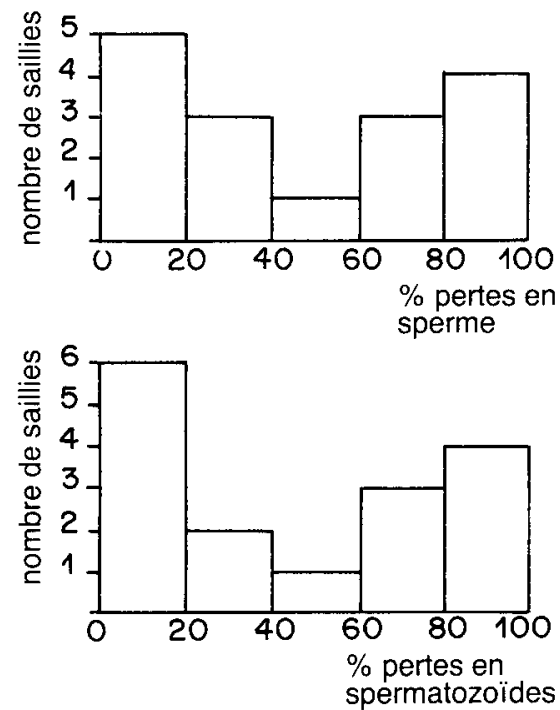
Tableau 4. Effet du mode d'éjaculation (collecte ou saillie) sur la production de spermatozoïdes $\left(\times 10^{9}\right)$ à différentes périodes d'éjaculation.

\begin{tabular}{|l|c|c|c|c|}
\hline \multirow{2}{*}{$\begin{array}{c}\text { Mode } \\
\text { d'éjaculation }\end{array}$} & \multicolumn{3}{|c|}{ Périodes d'éjaculation } & \multirow{2}{*}{ Total } \\
\cline { 2 - 5 } & (1) & (2) & (3) & \\
\hline Collecte & $\mathbf{6 3 , 1} \pm 14,1$ & $16,4 \pm 5,2$ & $2,6 \pm 1,1$ & $30,5 \pm 8,4$ \\
Saillie & $61,5 \pm 15,8$ & $14,8 \pm 3,5$ & $3,5 \pm 1,5$ & $30,1 \pm 8,7$ \\
\hline
\end{tabular}

(1) : $1^{\text {re }}$ éjaculation après 15 jours de repos sexuel. (2) : $4^{\mathrm{e}}$ éjaculation du rythme à 1 fois / jour.

(3) : $3^{\mathrm{e}}$ éjaculation au $4^{\mathrm{e}}$ jour du rythme à 4 fois / jour.

Tableau 5. Taux de gestation obtenus après accouplement naturel par des verrats soumis à différents rythmes de saillie.

\begin{tabular}{|c|c|c|c|c|c|c|}
\hline \multirow{3}{*}{$\begin{array}{c}\text { Jour } \\
\text { de l'accouplement }\end{array}$} & \multicolumn{6}{|c|}{ Fréquence journalière de saillie } \\
\hline & \multicolumn{3}{|c|}{1} & \multicolumn{3}{|c|}{4} \\
\hline & $\mathbf{1}$ & 4 & Total & 4 & 5 & Total \\
\hline $\begin{array}{l}\text { Nombre } \\
\text { de truies accouplées }\end{array}$ & 6 & 6 & 12 & 6 & 6 & 12 \\
\hline Taux de gestation (\%) & 83,3 & 100,0 & 91,6 & 50,0 & 66,6 & 58,3 \\
\hline $\begin{array}{l}\text { Taux de survie } \\
\text { embryonnaire (\%) }\end{array}$ & 71,6 & 67,3 & 69,2 & 79,5 & 79,1 & 79,2 \\
\hline
\end{tabular}

- accouplements de «bonne qualité » où il y a peu de perte en spermatozoïdes,

- accouplements de «mauvaise qualité » où les pertes sont importantes.

Si l'on tient compte de ces reflux, il n'apparaît aucune différence entre la production de sperme après accouplement ou après collecte (tableau 4).

\section{3 / Estimation de la fertilité}

Le taux de gestation obtenu lorsque le mâle n'effectue qu'un accouplement par jour, est supérieur à $90 \%$ et conforme en cela aux données de la bibliographie. Par contre, après plusieurs jours d'activité sexuelle intense seulement un peu plus de la moitié des truies sont fécondées $(58,3 \%)$. Le taux de mortalité embryonnaire n'est pas affecté (Tableau 5).

\section{3 / Discussion et conclusion}

L'accroissement du rythme d'activité sexuelle des verrats ne pose donc pas seulement un problème de fatigue dont pourrait se ressentir l'ardeur sexuelle du mâle, mais peut affecter directement la capacité de fécondation par insuffisance du nombre de spermatozoïdes.

Malgré une stimulation par une femelle, une proportion importante des verrats ne réagissent plus à la présentation du mannequin de collecte. En accouplement naturel, la stimulation par la femelle en chaleur, le changement de femelles au cours de saillies successives peut permettre d'accroître le nombre d'accouplements par rapport aux collectes. Toutefois, l'ardeur sexuelle du mâle peut devenir une limite à l'utilisation intensive des verrats.
Cependant, la composition de l'éjaculat est affectée bien avant que l'ardeur sexuelle ne soit modifiée. Ceci est encore aggravé par le phénomène de reflux de sperme. En effet, même après un repos sexuel nous avons constaté que, dès le quatrième accouplement, le nombre de spermatozoïdes pouvait descendre au-dessous de la limite nécessaire à une fécondation.

En pratique, il est donc nécessaire d'envisager une gestion de la production spermatique du mâle, même lorsque la reproduction s'effectue par accouplement naturel. La réduction du nombre de spermatozoïdes éjaculés, jointe aux pertes dues au reflux de sperme font que la fertilité peut être affectée, alors même que l'ardeur sexuelle persiste. La capacité de production spermatique du reproducteur est un facteur qui devrait alors être prise en compte.

Ce texte a été publié dans les Comptes-rendus des Journées de la Recherche porcine en France en 1985.

\section{Références bibliographiques}

DU MESNIL DU BUISSON F., SIGNORET J.P., 1970. Reproductive physiology and artificial insemination in pigs. Vet. Rec. 86, 562-568.

KAPLAN I., 1969. Effect of two collections per day on semen characters in boars. Zivocisna Vyroba. 14, 49-56.

SIGNORET J.P., DU MESNIL DU BUISSON F., BARITEAU F., 1971. L'insémination artificielle porcine. Bull. Tech. Inf., Minist. Agric. 257, 157-162.

SINGH G., 1962. Durée de passage dans l'épididyme des spermatozoïdes de verrat marquès au $\mathrm{P}_{32}$. Ann. Biol. Anim. Bioch. Bioph. 2, 43-46.

SWIERSTRA E.E., 1971. Sperm production of boars as measured from epididymal sperm reserves and quantitative testicular histology. J. Reprod. Fert. 27, 91-99. 